\title{
Value distribution of $q$-difference differential polynomials of entire functions
}

$\mathrm{Na}$ Xu, Ting-Bin Cao* and Kai Liu

\section{${ }^{*}$ Correspondence:}

tbcao@ncu.edu.cn

Department of Mathematics,

Nanchang University, Nanchang,

Jiangxi 330031, P.R. China

\begin{abstract}
For a complex value $q \neq 0,1$, and a transcendental entire function $f(z)$ with order, $0<\sigma(f)<\infty$, we study the value distribution of $q$-difference differential polynomials $\left[f^{n}(z)(f(q z)-f(z))\right]^{(k)}$ and $[f(z) f(q z)]^{(k)}$.
\end{abstract}

MSC: 30D35; 39A05

Keywords: Nevanlinna theory; q-difference; entire functions

\section{Introduction and main results}

A meromorphic function $f(z)$ means meromorphic in the complex plane $\mathbb{C}$. If no poles occur, then $f(z)$ reduces to an entire function. For every real number $x \geq 0$, we define $\log ^{+} x:=\max \{0, \log x\}$. Assume that $n(r, f)$ counts the number of poles of $f$ in $|z|<r$, each pole according to its multiplicity, and that $\bar{n}(r, f)$ counts the number of the distinct poles of $f$ in $|z|<r$, ignoring the multiplicity. The characteristic function of $f(z)$ is defined by

$$
T(r, f):=m(r, f)+N(r, f)
$$

where

$$
N(r, f):=\int_{0}^{r} \frac{n(t, f)-n(0, f)}{t} d t+n(0, f) \log r
$$

and

$$
m(r, f):=\frac{1}{2 \pi} \int_{0}^{2 \pi} \log ^{+}\left|f\left(r e^{i \theta}\right)\right| d \theta .
$$

The notation $\bar{N}(r, f)$ is similarly defined with $\bar{n}(r, f)$ instead of $n(r, f)$. More notations and definitions of the Nevanlinna value distribution theory of meromorphic functions can be found in $[1,2]$.

A meromorphic function $\alpha(z)$ is called a small function with respect to $f(z)$, if $T(r, \alpha)=$ $S(r, f)$, where $S(r, f)$ denotes any quantity satisfying $S(r, f)=o(T(r, f))$ as $r \rightarrow \infty$ outside a possible exceptional set $E$ of finite logarithmic measure. The order and the exponent of convergence of zeros of meromorphic function $f(z)$ is, respectively, defined as

$$
\sigma(f)=\limsup _{r \rightarrow \infty} \frac{\log T(r, f)}{\log r}, \quad \lambda(f)=\limsup _{r \rightarrow \infty} \frac{\log N\left(r, \frac{1}{f}\right)}{\log r} .
$$

@2014 Xu et al.; licensee Springer. This is an Open Access article distributed under the terms of the Creative Commons Attribution License (http://creativecommons.org/licenses/by/2.0), which permits unrestricted use, distribution, and reproduction in any medium, provided the original work is properly cited. 
The difference operators for a meromorphic function $f(z)$ are defined as

$$
\begin{aligned}
& \Delta_{c} f(z)=f(z+c)-f(z) \quad(c \neq 0), \\
& \nabla_{q} f(z)=f(q z)-f(z) \quad(q \neq 0,1) .
\end{aligned}
$$

A Borel exceptional value of $f(z)$ is any value $a \in \mathbb{C} \cup\{\infty\}$ satisfying $\lambda(f-a)<\sigma(f)$, where $\lambda(f-\infty)$ means $\lambda\left(\frac{1}{f}\right)$.

Recently, the difference variant of the Nevanlinna theory has been established independently in [3-6]. Using these theories, value distributions of difference polynomials have been studied by many papers. For example, Laine and Yang [7] proved that if $f(z)$ is a transcendental entire function of finite order, $c$ is a nonzero complex constant and $n \geq 2$, then $f^{n}(z) f(z+c)$ takes every nonzero value infinitely often.

Chen [8] considered the value distribution of $f(z) f(z+c)$ and obtained the following theorem.

Theorem A [8, Corollary 1.3] Let $f(z)$ be a transcendental entire function of finite order, and let $c$ be a nonzero complex constant. If $f(z)$ has the Borel exceptional value 0 , then $H(z)=f(z) f(z+c)$ takes every nonzero value $a \in \mathbb{C}$ infinitely often.

Chen [9] considered zeros of difference product $H_{n}(z)=f^{n}(z) \Delta_{c} f(z)$ and gave some conditions that guarantee $H_{n}(z)$ has finitely many zeros or infinitely many zeros.

Theorem B [9, Theorem 1] Let $f(z)$ be a transcendental entire function of finite order and $c \in \mathbb{C} \backslash\{0\}$ be a constant such that $f(z+c) \not \equiv f(z)$. Set $H_{n}(z)=f^{n}(z) \Delta_{c} f(z)$ where $\Delta_{c} f(z)=$ $f(z+c)-f(z), n \geq 2$ is an integer. Then the following statements hold.

(1) If $f(z)$ satisfies $\sigma(f) \neq 1$, or has infinitely many zeros, then $H_{n}(z)$ has infinitely many zeros.

(2) Iff $(z)$ has only finitely many zeros and $\sigma(f)=1$, then $H_{n}(z)$ has only finitely many zeros.

The zero distribution of differential polynomials is a classical topic in the theory of meromorphic functions. Hayman [10, Theorem 10] firstly considered the value distribution of $f^{n} f^{\prime}-1$, where $f$ is a transcendental function.

Recently, Liu, Liu and Cao [11] investigated the zeros of $\left[f(z)^{n} f(z+c)\right]^{(k)}-\alpha(z)$ and $\left[f(z)^{n} \Delta_{c} f(z)\right]^{(k)}-\alpha(z)$, where $\alpha(z)$ is a nonzero small function with respect to $f(z)$.

Theorem C [11, Theorems 1.1 and 1.3] Let $f(z)$ be a transcendental entire function of finite order and $\alpha(z)$ be a nonzero small function with respect to $f(z)$. If $n \geq k+2$, then $\left[f(z)^{n} f(z+\right.$ $c)]^{(k)}-\alpha(z)$ has infinitely many zeros. If $f(z)$ is not a periodic function with period $c$ and $n \geq k+3$, then $\left[f(z)^{n} \Delta_{c} f(z)\right]^{(k)}-\alpha(z)$ has infinitely many zeros.

The main purpose of this paper is to consider a transcendental entire function $f(z)$ with positive and finite order and obtain some results on the value distributions of the $q$-difference differential polynomials $\left[f^{n}(z)(f(q z)-f(z))\right]^{(k)}$ and $[f(z) f(q z)]^{(k)}$. The first theorem will consider what conditions guarantee that $\left[f^{n}(z) \nabla_{q} f(z)\right]^{(k)}$ has infinitely many zeros. 
Theorem 1.1 Let $f(z)$ be a transcendental entire function of finite and positive order $\sigma(f)$, $q \in \mathbb{C} \backslash\{0,1\}$ be a constant such that $q^{\sigma(f)} \neq 1$ and $f(z) \not \equiv f(q z)$. Set $H(z)=\left[f^{n}(z) \nabla_{q} f(z)\right]^{(k)}$, $n \geq 1$ is an integer. If $f(z)$ has finitely many zeros, then $H(z)-\alpha(z)$ has infinitely many zeros, where $\alpha(z)$ is a nonzero small entire function with respect to $f(z)$.

In the following, we will study the value distribution of $[f(z) f(q z)]^{(k)}$.

Theorem 1.2 Let $f(z)$ be a transcendental entire function of finite and positive order $\sigma(f)$, a be a finite Borel exceptional value of $f(z), q \in \mathbb{C} \backslash\{0,1\}$ be a constant such that $q^{\sigma(f)} \neq \pm 1$. Set $H(z)=[f(z) f(q z)]^{(k)}$, then the following statements hold.

(1) If $a=0$, then 0 is also the Borel exceptional value of $H(z)$. So that $H(z)$ has no nonzero finite Borel exceptional value.

(2) If $a \neq 0$, then $H(z)$ has no finite Borel exceptional value.

(3) $H(z)$ takes every nonzero value $c \in \mathbb{C}$ infinitely often and satisfies $\lambda(H-c)=\sigma(f)$.

Using the similar method of the proof of Theorem 1.2(1), we get the following result immediately.

Corollary 1.1 Let $f(z)$ be a transcendental entire function of finite and positive order $\sigma(f)$, $q \in \mathbb{C} \backslash\{0,1\}$ be a constant such that $q^{\sigma(f)} \neq-n$. If 0 is a Borel exceptional value of $f(z)$, then 0 is also the Borel exceptional value of $\left[f^{n}(z) f(q z)\right]^{(k)}$.

\section{Some lemmas}

The following are the well-known Weierstrass factorization and Hadamard factorization theorems.

Lemma 2.1 [12] If an entire function $f(z)$ has a finite exponent of convergence $\lambda(f)$ for its zero-sequence, then $f(z)$ has a representation in the form

$$
f(z)=Q(z) e^{g(z)},
$$

satisfying $\lambda(Q)=\sigma(Q)=\lambda(f)$. Further, if $f(z)$ is of finite order, then $g(z)$ in the above form is a polynomial of degree less or equal to the order of $f(z)$.

Lemma 2.2 [13] Suppose that $f_{1}(z), f_{2}(z), \ldots, f_{n}(z)(n \geq 2)$ are meromorphic functions and $g_{1}(z), g_{2}(z), \ldots, g_{n}(z)$ are entire functions satisfying the following conditions:

(1) $\sum_{j=1}^{n} f_{j}(z) e^{g_{j}(z)} \equiv 0$;

(2) $g_{j}(z)-g_{k}(z)$ are not constants for $1 \leq j<k \leq n$;

(3) For $1 \leq j \leq n, 1 \leq h<k \leq n, T\left(r, f_{j}\right)=o\left(T\left(r, e^{g_{h}-g_{k}}\right)\right)(r \rightarrow \infty, r \notin E)$.

Then $f_{j}(z) \equiv 0(j=1,2, \ldots, n)$.

\section{The proofs}

\subsection{Proof of Theorem 1.1}

Since $f(z)$ is a transcendental entire function of finite order and has finitely many zeros, then by Lemma 2.1, $f(z)$ can be written as

$$
f(z)=g(z) e^{h(z)}
$$


where $g(z)(\not \equiv 0), h(z)$ are polynomials. Set

$$
h(z)=a_{s} z^{s}+\cdots+a_{0},
$$

where $a_{s}, a_{s-1}, \ldots, a_{0}$ are constants, and $a_{s} \neq 0$. Since $\sigma(f) \neq 0$, then $\sigma(f)=\operatorname{deg}(h(z))=s \geq 1$. So

$$
\begin{aligned}
H(z) & =\left[f^{n}(z) \nabla_{q} f(z)\right]^{(k)} \\
& =\left[g^{n}(z) g(q z) e^{n h(z)+h(q z)}-g^{n+1}(z) e^{(n+1) h(z)}\right]^{(k)} \\
& =g_{1}(z) e^{n h(z)+h(q z)}-g_{2}(z) e^{(n+1) h(z)},
\end{aligned}
$$

where $g_{1}(z), g_{2}(z)$ are nonzero polynomials and

$$
(n+1) h(z)-(n h(z)+h(q z))=\left(1-q^{s}\right) a_{s} z^{s}+\left(1-q^{s-1}\right) a_{s-1} z^{s-1}+\cdots+(1-q) a_{1} z
$$

Since $q^{\sigma(f)}=q^{s} \neq 1$, then $(n+1) h(z)-(n h(z)+h(q z))$ is not a constant. So $H(z)$ is a transcendental entire function, suppose that $H(z)-\alpha(z)$ has finitely many zeros, then by Lemma 2.1, $H(z)-\alpha(z)$ can be written as

$$
H(z)-\alpha(z)=g_{3}(z) e^{h_{1}(z)} .
$$

Here $g_{3}(z)(\not \equiv 0), h_{1}(z)$ are polynomials. Combing the above equalities, we obtain

$$
g_{1}(z) e^{n h(z)+h(q z)}-g_{2}(z) e^{(n+1) h(z)}-g_{3}(z) e^{h_{1}(z)}-\alpha(z)=0 .
$$

Note that $(n+1) h(z)-(n h(z)+h(q z))$ is not a constant and $g_{1}(z), g_{2}(z), g_{3}(z)$ are nonzero polynomials. If $(n h(z)+h(q z))-h_{1}(z)$ and $(n+1) h(z)-h_{1}(z)$ are not constants, then by (3.1) and Lemma 2.2, we obtain

$$
g_{1}(z) \equiv 0, \quad g_{2}(z) \equiv 0, \quad g_{3}(z) \equiv 0, \quad \alpha(z) \equiv 0 .
$$

This is a contradiction.

If $(n h(z)+h(q z))-h_{1}(z)=c$, where $c$ is a constant, then (3.1) can be rewritten as

$$
\left(g_{1}(z)-e^{-c} g_{3}(z)\right) e^{n h(z)+h(q z)}-g_{2}(z) e^{(n+1) h(z)}-\alpha(z)=0 .
$$

By (3.2) and Lemma 2.2, we obtain

$$
g_{1}(z)-e^{-c} g_{3}(z) \equiv 0, \quad g_{2}(z) \equiv 0, \quad \alpha(z) \equiv 0 .
$$

This is a contradiction.

If $(n+1) h(z)-h_{1}(z)=c$, where $c$ is a constant, then using the same method as above, we also obtain a contradiction.

Hence $H(z)-\alpha(z)$ has infinitely many zeros. 


\subsection{Proof of Theorem 1.2}

Since $f(z)$ is a transcendental entire function of finite and positive order with a Borel exceptional value $a$, then by Lemma $2.1, f(z)$ can be written as

$$
f(z)=a+g(z) e^{\alpha z^{s}}
$$

Here $s$ is a positive integer, $\alpha$ is a nonzero constant, $g(z)$ is a nonzero entire function satisfying $\sigma(g)<\sigma(f)=s$, thus

$$
\begin{aligned}
f(z) f(q z) & =\left(a+g(z) e^{\alpha z^{s}}\right)\left(a+g(q z) e^{\alpha q^{s} z^{s}}\right) \\
& =a^{2}+a g(q z) e^{\alpha q^{s} z^{s}}+a g(z) e^{\alpha z^{s}}+g(z) g(q z) e^{\alpha\left(1+q^{s}\right) z^{s}} .
\end{aligned}
$$

Since $q^{\sigma(f)}=q^{s} \neq \pm 1, g(z)$ is a nonzero entire function satisfying $\sigma(g)<s, f(z) f(q z)$ is a transcendental entire function and $\sigma(f(z) f(q z))=s$, and by the classical result of Nevanlinna theory, we get $\sigma(H)=\sigma(f(z) f(q z))=s$. Then

$$
\begin{aligned}
H(z) & =[f(z) f(q z)]^{(k)} \\
& =\left[a^{2}+a g(q z) e^{\alpha q^{s} z^{s}}+a g(z) e^{\alpha z^{s}}+g(z) g(q z) e^{\alpha\left(1+q^{s}\right) z^{s}}\right]^{(k)} \\
& =g_{1}(z) e^{\alpha q^{s} z^{s}}+g_{2}(z) e^{\alpha z^{s}}+g_{3}(z) e^{\alpha\left(1+q^{s}\right) z^{s}} .
\end{aligned}
$$

Here $g_{1}(z), g_{2}(z), g_{3}(z)$ are differential polynomials of $g(z), g(q z)$ and $\sigma\left(g_{i}(z)\right)<s, i=1,2,3$.

Case 1. $a=0$, by the above equality, we get

$$
H(z)=g_{3}(z) e^{\alpha\left(1+q^{s}\right) z^{s}}
$$

Since $q^{\sigma(f)}=q^{s} \neq-1$ and $\sigma\left(g_{3}(z)\right)<s$, this implies that 0 is the Borel exceptional value of $H(z)$.

Case 2. $a \neq 0$, suppose $H(z)$ has a finite Borel exceptional value $b$, then by Lemma 2.1, $H(z)$ can be written as

$$
H(z)=b+h(z) e^{\beta z^{s}} .
$$

Here $\beta$ is a nonzero constant, $h(z)$ is a nonzero entire function satisfying $\sigma(h)<\sigma(H)=s$. Combing the above equalities, we obtain

$$
g_{1}(z) e^{\alpha q^{s} z^{s}}+g_{2}(z) e^{\alpha z^{s}}+g_{3}(z) e^{\alpha\left(1+q^{s}\right) z^{s}}-h(z) e^{\beta z^{s}}-b=0 .
$$

Since $q^{\sigma(f)}=q^{s} \neq \pm 1$, we have $\alpha q^{s} \neq \alpha \neq \alpha\left(1+q^{s}\right)$.

If $\alpha q^{s} \neq \beta, \alpha \neq \beta, \alpha\left(1+q^{s}\right) \neq \beta$. By (3.3) and Lemma 2.2, we obtain

$$
g_{1}(z) \equiv 0, \quad g_{2}(z) \equiv 0, \quad g_{3}(z) \equiv 0, \quad h(z) \equiv 0, \quad b \equiv 0 .
$$

This is a contradiction.

If $\alpha q^{s}=\beta$, then (3.3) can be rewritten as

$$
\left(g_{1}(z)-h(z)\right) e^{\alpha q^{s} z^{s}}+g_{2}(z) e^{\alpha z^{s}}+g_{3}(z) e^{\alpha\left(1+q^{s}\right) z^{s}}-b=0 .
$$


By (3.4) and Lemma 2.2, we obtain

$$
g_{1}(z)-h(z) \equiv 0, \quad g_{2}(z) \equiv 0, \quad g_{3}(z) \equiv 0, \quad b \equiv 0 .
$$

Which is a contradiction.

If $\alpha=\beta$ or $\alpha\left(1+q^{s}\right)=\beta$, then using the same method as above, we also obtain a contradiction.

Case 3. From Case 1 and Case 2, we get that if $f(z)$ has a finite Borel exceptional value, then any nonzero finite value $c$ must not be the Borel exceptional value of $H(z)$, so $H(z)$ takes every nonzero value $c \in \mathbb{C}$ infinitely often, since $\sigma(H)=s$, then $\lambda(H-c)=\sigma(H)=$ $\sigma(f)$.

The proof of Theorem 1.2 is completed.

Competing interests

The authors declare that they have no competing interests.

Authors' contributions

All authors drafted the manuscript, read and approved the final manuscript.

\section{Acknowledgements}

This research was partly supported by the NSFC (no. 11101201, 11301260), Foundation of Post Ph.D. of Jiangxi, the NSF of Jiangxi (no. 20122BAB211001, 20132BAB211003) and NSF of education department of Jiangxi (no. GJJ13077, GJJ13078) of China.

Received: 26 September 2013 Accepted: 18 February 2014 Published: 07 Mar 2014

\section{References}

1. Hayman, W: Meromorphic Functions. Clarendon, Oxford (1964)

2. Yang, L: Value Distribution Theory. Science Press, Beijing (1982). Springer, Berlin (1993)

3. Barnett, DC, Halburd, RG, Korhonen, RJ, Morgan, W: Nevanlinna theory for the q-difference operator and meromorphic solutions of q-difference equations. Proc. R. Soc. Edinb., Sect. A 137, 457-474 (2007)

4. Chiang, YM, Feng, SJ: On the Nevanlinna characteristic $f(z+\eta)$ and difference equations in the complex plane. Ramanujan J. 16, 105-129 (2008)

5. Halburd, RG, Korhonen, RJ: Nevanlinna theory for the difference operator. Ann. Acad. Sci. Fenn., Math. 31, 463-478 (2006)

6. Halburd, RG, Korhonen, RJ: Difference analogue of the lemma on the logarithmic derivative with applications to difference equations. J. Math. Anal. Appl. 314, 477-487 (2006)

7. Laine, I, Yang, CC: Value distribution of difference polynomials. Proc. Jpn. Acad., Ser. A, Math. Sci. 83, 148-151 (2007)

8. Chen, ZX, Huang, ZB, Zheng, XM: On properties of difference polynomials. Acta Math. Sci. 31B(2), 627-633 (2011)

9. Chen, ZX: Value distribution of products of meromorphic functions and their differences. Taiwan. J. Math. 15, 1411-1421 (2011)

10. Hayman, WK: Picard values of meromorphic functions and their derivatives. Ann. Math. 70, 9-42 (1959)

11. Liu, K, Liu, XL, Cao, TB: Some results on zeros and uniqueness of difference-differential polynomials. Appl. Math. J. Chin. Univ. Ser. B 27, 94-104 (2012)

12. Ash, R: Complex Variables. Academic Press, New York (1971)

13. Yi, HX, Yang, CC: Uniqueness Theory of Meromorphic Functions. Science Press, Beijing (1995). Kluwer Academic, Dordrecht (2003)

10.1186/1687-1847-2014-80

Cite this article as: Xu et al.: Value distribution of $q$-difference differential polynomials of entire functions. Advances in Difference Equations 2014, 2014:80 